\title{
Increased prevalence of anemia in pediatric patients with severe asthma.
}

\author{
Ji-Eun Chang ${ }^{1}$ and Kiyon Rhew ${ }^{2}$ \\ ${ }^{1}$ Dongduk Women's University \\ ${ }^{2}$ Dongduk Women's University
}

August 23, 2020

Chronic inflammatory diseases cause anemia of inflammation (AI). This causes impaired immune-mediated regulation of hepcidin and several cytokines, which play a major role in iron homeostasis, accelerates turnover of red blood cells, and affects the activity of erythropoietin (EPO), thereby reducing levels of hemoglobin and iron. ${ }^{1}$ Asthma is one of common allergic diseases caused by disturbance of the immune system, ${ }^{2}$ and this chronic inflammatory condition could cause systemic inflammation. ${ }^{3}$ Several researches have recently shown that asthma association with the increased prevalence of anemia. ${ }^{4,5}$ No studies, however, have been conducted on whether severe or uncontrolled asthma can more increase the prevalence of anemia.

We hypothesized that patients with severe asthma were more likely to have an active inflammatory condition, which may increase the prevalence of anemia. Therefore, this study aims to evaluate whether the prevalence of anemia in pediatric asthma patients differs according to asthma control or severity.

$98 \%$ of Koreans are enrolled in the universal health insurance system, and all medical information is reported to the Health Insurance Review Agency (HIRA). In this study, $10 \%\left({ }^{\sim} 1,100,000\right)$ of patients under the age of 20 provided by HIRA were analyzed using a dataset constructed by random stratification based on age and sex (HIRA-PPS-2016). ${ }^{6}$ We included patients younger than 18 years and patients prescribed asthma medications with diagnosis of asthma (eTable1, eTable2). Patients diagnosed with anemia other than iron deficiency anemia (IDA) or AI were excluded. We classified the severe asthma group who were diagnosed with asthma exacerbation at least once a year or asthma diagnosis with systemic steroids, and the rest were defined as the non-severe asthma group. Binary logistic regression was carried out by applying inverse probability of treatment weighting (IPTW) using the propensity score. To calculate the predicting propensity score, we used a multivariable logistic regression model to estimate the probability of each patient, including confounders (eTable1). Odds ratio (OR) with $95 \%$ confidence interval (CI) are reported for association anemia and asthma severity.

A total of 236,429 pediatric patients were included in the study. The flow diagram for the study subject inclusion is shown (Fig. 1) and the characteristics of the two groups of patients were similarly adjusted after applying IPTW (eTable3). The increased prevalence of anemia in patients with severe asthma was expressed OR of 1.56 (95\% CI, 1.49-1.64; $\mathrm{P}<.001$ ) (Table 1). The association between anemia and asthma severity in children, male, female, or patients under health insurance was expressed OR of 1.63 (95\% CI, 1.55-1.71; P $<.001$ ), 1.59 (95\% CI, 1.49-1.70; P <.001), 1.53 (95\% CI, 1.42-1.64; P <.001), and 1.56 (95\% CI, 1.49-1.64; $\mathrm{P}<.001)$, respectively. There were no associations between anemia and severe asthma in adolescents and patients under medical aid (Table 1).

The prevalence of anemia in patients who experienced exacerbation of asthma or with systemic steroid was analyzed higher than other asthma patients. This was the same even after adjusting for other confounding factors in the two patient groups. Previous study suggested that the correlation between the two diseases 
in those groups may have been insignificant since adolescent patients or patients under medical aid program may have different medical service usage patterns from those of general patients. ${ }^{5}$

We do not monitor anemia for asthma patients in general, and the guidelines do not present it in this regard. However, anemia negatively affects pediatric patients' growth, cognitive abnormalities, and poor quality of life. ${ }^{7,8}$ In addition, in the case of AI, treatment or control of the underlying disease takes precedence over the treatment of anemia rather than simply administering iron or EPO. ${ }^{9}$ In addition, it was reported that in patients with high hepcidin, an index that can distinguish between AI and IDA, administration of EPO or iron administration was less or non-response to the treatment of anemia. ${ }^{11-12}$ Therefore, it might be recommended to monitor and treat anemia since the prevalence of anemia may increase in pediatric patients with severe asthma.

This study has some limitations. First, we reported the association between severe asthma and anemia which is not the causality. Secondly, since we included patients diagnosed with IDA, the influence of patients with isolated IDA cannot be ruled out. Nevertheless, our study has several strengths including a large sample size and statistical adjustments with propensity weighting.

To our knowledge, this is the first study to show that severe asthma has a stronger association with anemia compared to non-severe or controlled asthma. This suggests that it may be necessary to more closely monitor anemia in patients with severe asthma. Further study is needed to better understand the etiology that severe asthma causes more AI.

\section{References}

1. Zarychanski R and Houston DS. Anemia of chronic disease: a harmful disorder or an adaptive, beneficial response? CMAJ. 2008;179(4):333-7.

2. Thomsen SF. Epidemiology and natural history of atopic diseases. Eur Clin Respir J. 2015;2:10.

3. Wouters EFM, Reynaert NL, Dentener MA, et al. Systemic and Local Inflammation in Asthma and Chronic Obstructive Pulmonary Disease: Is There a Connection? Proc Am Thorac Soc. 2009;6:638-47.

4. Drury KE, Schaeffer M, Silverberg JI. Association Between Atopic Disease and Anemia in US Children. JAMA Pediatr. 2016;170:29-34.

5. Rhew K, Brown JD, Oh JM. Atopic Disease and Anemia in Korean Patients: Cross-Sectional Study with Propensity Score Analysis. Int J Environ Res Public Health. 2020;17:1978

6. Kim L, Kim JA, Kim S. A guide for the utilization of Health Insurance Review and Assessment Service National Patient Samples. Epidemiol Health. 2014;36:e2014008.

7. Allali S, Brousse V, Sacri AS, et al. Anemia in children: prevalence, causes, diagnostic work-up, and long-term consequences. Expert Rev Hematol. 2017;10:1023-8.

8. Milman N.Iron prophylaxis in pregnancy - General or individual and in which dose? Ann Hematol. 2006;85:821-8.

9. Nairz M, Weiss G. Infections Associated with Iron Administration. Met Ions Life Sci. 2019;19

10. Poggiali E, De Amicis MM, Motta I. Anemia of chronic disease: a unique defect of iron recycling for many different chronic diseases. Eur J Intern Med. 2014;25:12-7.

11. Theurl M, Nairz M, Schroll A, et al. aHepcidin as a predictive factor and therapeutic target in erythropoiesis-stimulating agent treatment for anemia of chronic disease in rats. Haematologica. 2014;99:1516-24.

12. Bregman DB, Morris D, Koch TA, et al. Hepcidin levels predict nonresponsiveness to oral iron therapy in patients with iron deficiency anemia. Am J Hematol. 2013;88:97-101. 


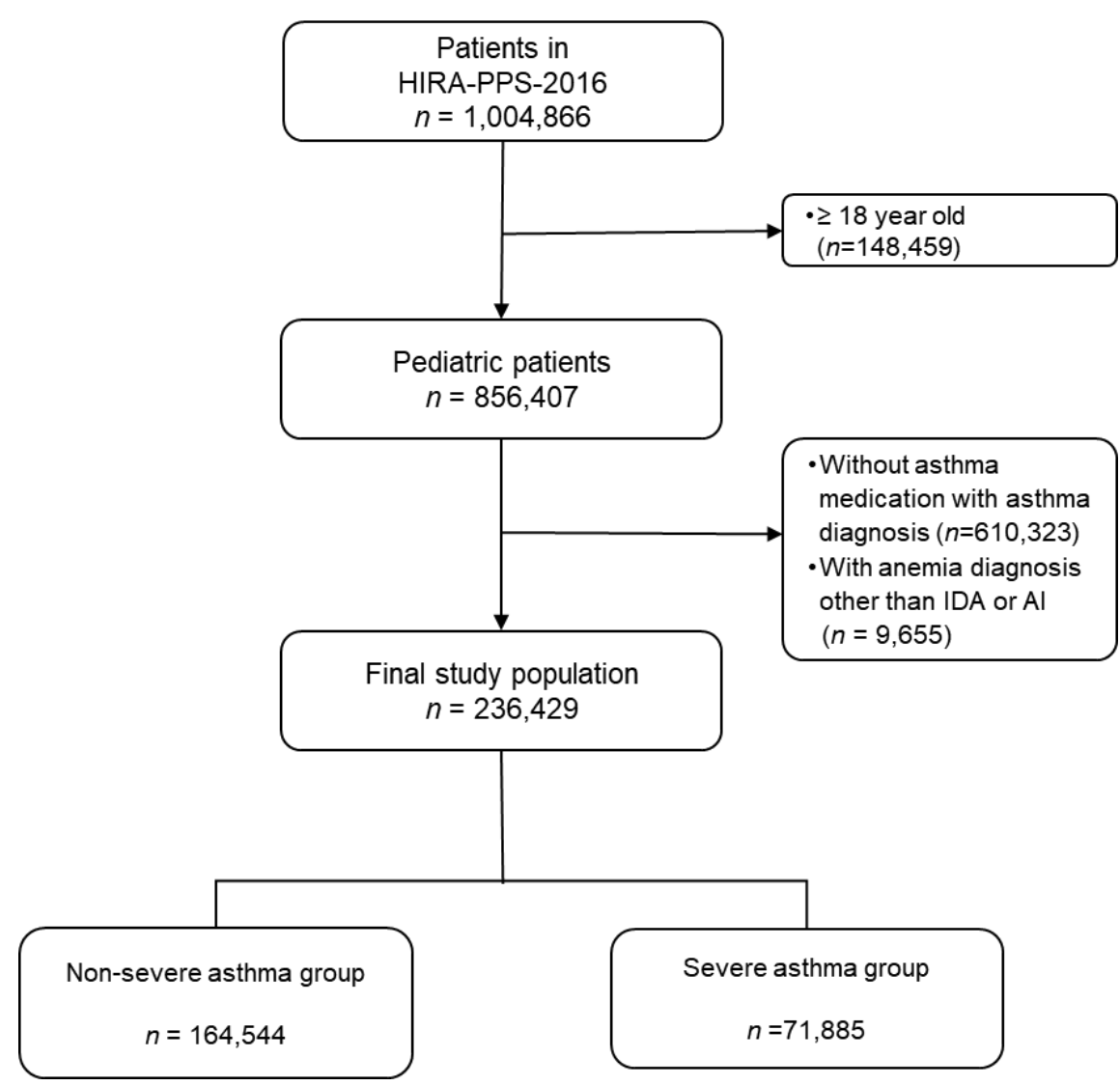

Fig. 1 Flow diagram for study subject inclusion

HIRA: Health Insurance Review Agency, IDA: iron deficiency anemia, AI: anemia of inflammation

Table 1. Association between asthma severity and anemia in pediatric patients after adjusting with propensity weighting.

\begin{tabular}{|c|c|c|c|c|}
\hline Characteristics of patients & Characteristics of patients & Asthma severity & Odds ratio $(95 \% \mathrm{CI})$ & $\mathrm{P}$ value \\
\hline \multirow[t]{2}{*}{ All patients } & \multirow[t]{2}{*}{ All patients } & Non-severe asthma & 1 [Reference] & \\
\hline & & Severe asthma & $1.56(1.49-1.64)$ & $<.001$ \\
\hline Subgroup analysis & Subgroup analysis & & & \\
\hline \multirow[t]{4}{*}{ Age } & \multirow[t]{2}{*}{ Children (<12 years) } & Non-severe asthma & 1 [Reference] & \\
\hline & & Severe asthma & $1.63(1.55-1.71)$ & $<.001$ \\
\hline & \multirow[t]{2}{*}{ Adolescents ([?]12, <18 years) } & Non-severe asthma & 1 [Reference] & \\
\hline & & Severe asthma & $1.0090 .81-1.23)$ & 0.970 \\
\hline \multirow[t]{4}{*}{ Gender } & \multirow[t]{2}{*}{ Male } & Non-severe asthma & 1 [Reference] & \\
\hline & & Severe asthma & $1.59(1.49-1.70)$ & $<.001$ \\
\hline & \multirow[t]{2}{*}{ Female } & Non-severe asthma & 1 [Reference] & \\
\hline & & Severe asthma & $1.53(1.42-1.64)$ & $<.001$ \\
\hline \multirow[t]{4}{*}{ Insurance type } & \multirow[t]{2}{*}{ Health insurance } & Non-severe asthma & 1 [Reference] & \\
\hline & & Severe asthma & $1.56(1.49-1.64)$ & $<.001$ \\
\hline & \multirow[t]{2}{*}{ Medical aid } & Non-severe asthma & 1 [Reference] & \\
\hline & & Severe asthma & $1.45(0.99-2.12)$ & 0.057 \\
\hline
\end{tabular}


eTable 1. The list of KCD-7 diagnostic codes of diseases in the study.

eTable 2. The list of ATC codes of asthma medication in the study.

eTable 3. Baseline characteristics of subjects before and after inverse probability of treatment weighting

\section{Conflict of Interest}

The authors declared no potential conflicts of interest with respect to the research, authorship, and/or publication of this article.

\section{Authorship}

K.R. defined the research question, and designed the method. J.C analyzed the data and wrote the first manuscript. K.R. interpreted the results. K.R. reviewed and edited the paper. All authors approved the manuscript.

\section{Authors:}

Kiyon Rhew ${ }^{1}$ and Ji-Eun Chang ${ }^{1}$

${ }^{1}$ College of Pharmacy, Dongduk Women's University, 60 Hwarang-ro 13-gil, Seongbuk-gu, Seoul 02748, Republic of Korea.

\section{Corresponding Author:}

Kiyon Rhew,

College of Pharmacy, Dongduk Women's University, 60 Hwarang-ro 13-gil, Seongbuk-gu, Seoul, Republic of Korea, 02748

Tel: $82-2-940-4519$

E-mail:kiyon@dongduk.ac.kr 